\title{
Broadband polarization degeneracy of guided waves in subwavelength structured $\mathrm{ZnO}$ pattern
}

Yermakov, Oleh Y.; Bogdanov, Andrey A.; Lavrinenko, Andrei

Published in:

IEEE Journal of Selected Topics in Quantum Electronics

Link to article, DOI:

10.1109/JSTQE.2018.2886306

Publication date:

2019

Document Version

Peer reviewed version

Link back to DTU Orbit

Citation $(A P A)$ :

Yermakov, O. Y., Bogdanov, A. A., \& Lavrinenko, A. (2019). Broadband polarization degeneracy of guided waves in subwavelength structured ZnO pattern. IEEE Journal of Selected Topics in Quantum Electronics, 25(3), [8200307]. https://doi.org/10.1109/JSTQE.2018.2886306

\section{General rights}

Copyright and moral rights for the publications made accessible in the public portal are retained by the authors and/or other copyright owners and it is a condition of accessing publications that users recognise and abide by the legal requirements associated with these rights.

- Users may download and print one copy of any publication from the public portal for the purpose of private study or research.

- You may not further distribute the material or use it for any profit-making activity or commercial gain

- You may freely distribute the URL identifying the publication in the public portal 


\title{
Broadband Polarization Degeneracy of Guided Waves in Subwavelength Structured ZnO Pattern
}

\author{
Oleh Y. Yermakov (), Student Member, IEEE, Andrey A. Bogdanov (i), and Andrei V. Lavrinenko (i)
}

(Invited Paper)

\begin{abstract}
Polarization degeneracy of electromagnetic plane waves in vacuum and in any bulk isotropic media is the keynote operational principle of many optical devices such as polarizers and interferometers. However, surface and guided waves spectra are typically either not degenerated at all or meet degeneracy only at very specific dispersion points. In this paper, we offer a design of a periodic photonic structure based on zinc oxide $(\mathrm{ZnO})$ nanocylinders providing the broadband polarization degeneracy of the guided waves in the near-IR frequency range. We analyze the impact of the spatial dispersion and substrate on the degeneracy breaking. We offer the design based on $\mathrm{ZnO}$ nanocylinders with aluminum-doped zinc oxide substrate, achieving the degeneracy in the vicinity of a telecommunication wavelength, for the practical implementation. Finally, we propose and verify numerically a potentially important device-waveguide polarizer, which is the analogue to the $\lambda / 4$ plate for the guided waves.
\end{abstract}

Index Terms-Zinc oxide, photonic crystal waveguide, guided waves, degeneracy, polarization, dispersion, telecom, nonlocality, aluminum-doped zinc oxide.

\section{INTRODUCTION}

A LL-DIELECTRIC materials have recently become one of the main platforms in the nanophotonics due to their inimitable advantages such as low losses and high refractive index in the optical range and relatively simple fabrication process [1], [2]. Another important feature is a manifestation of the magnetic response of a resonant high-index dielectric nanoparticle in the visible and mid-IR frequency ranges [3], [4]. The interplay between Mie resonances of the dielectric nanoparticles paves a way to the nanostructures with extraordinary optical properties [5], [6], for instance, suppression of backward or forward scattering (optical nanoantennas [7], Huygens' metasurfaces [8], [9]), enhanced Purcell factor (optical nanoantennas [7], [10]), total

Manuscript received July 31, 2018; revised November 21, 2018; accepted December 4, 2018. Date of publication December 11, 2018; date of current version December 28, 2018. This work was supported in part by the Russian Foundation for Basic Research under Grants 17-02-01234 and 18-32-00739 and in part by the Ministry of Education and Science of the Russian Federation under Grant 3.1668.2017/4.6. The work of O. Y. Yermakov and A. Bogdanov was supported by the Foundation for the Advancement of Theoretical Physics and Mathematics "BASIS". (Corresponding author: Oleh Y. Yermakov.)

O. Yermakov and A. Bogdanov are with the Department of Nanophotonics and Metamaterials, ITMO University, Saint Petersburg 197101, Russia (e-mail: o.yermakov@metalab.ifmo.ru; a.bogdanov@metalab.ifmo.ru).

A. Lavrinenko is with the DTU Fotonik, Technical University of Denmark, DK-2800 Kongens Lyngby, Denmark (e-mail: alav@ fotonik.dtu.dk).

Color versions of one or more of the figures in this paper are available online at http://ieeexplore.ieee.org.

Digital Object Identifier 10.1109/JSTQE.2018.2886306 radiation suppression (anapole mode [11], [12]), polarization control of light at nanoscale [13], [14]. Besides, all-dielectric structures have significantly extended the opportunities to manipulate surface waves, namely Dyakonov surface waves, in anisotropic media [1], [15], [16].

It is worth noting that modern technologies assist the fabrication of the extremely precise, single-crystalline, sub-micron nanoparticles [2]. The determination of a specific material plays an important role for the further investigation. For this work we chose zinc oxide $(\mathrm{ZnO})$ - a crucial technological material possessing numerous attractive characteristics for the optoelectronic and spintronic devices [17], photovoltaics [18] and sensing [19]. ZnO is a low-cost direct wide bandgap semiconductor (but in this work we consider it as the dielectric material) which has a number of advantages for the growth, synthesis and fabrication [20]. For example, zinc oxide can be grown on a substrate, such as glass, at low temperatures [17]. Nanostructured $\mathrm{ZnO}$ materials, such as nanowires, nanorods and nanoparticles, attracted attention due to their simplicity and prominent optical properties [20]. For instance, laser ablation in superfluid helium of $\mathrm{ZnO}$ allows to produce spheres with typical sizes of several tens of nanometers [21].

It is well-known that the light waves spectrum in free space is double degenerated with respect to the polarization degrees of freedom. One of the possible eigenmodes bases can be chosen with right- and left-handed circularly polarized plane waves. Many classical bulk optical devices, such as polarizers and interferometers, are based on the degeneracy breaking due to the birefringence. However, surface and waveguide modes are usually degenerated only for single fixed frequencies where the accidental degeneracy takes place due to the band crossing [22] [24]. It significantly limits the development of flat optics devices. For instance, surface analogues of polarization devices cannot be implemented due to the lack of the polarization degeneracy of surface waves. If it can be achieved this will make a pronounced step towards the polarization switching, strong spin-orbit interactions and unprecedented wavefront control. In addition, degenerated TE and TM surface waves form a promising platform for the efficient silicon-on-insulator-based coupling [25]-[27] and surface-enhanced chiral sensing [28], [29].

Huygens-like dielectric metasurfaces support near-unity transmission with full phase control in the visible [9], [30], [31] and radiofrequency ranges [8], [32], [33] opening new routes for the wavefront manipulation. The efficient beam steering with 

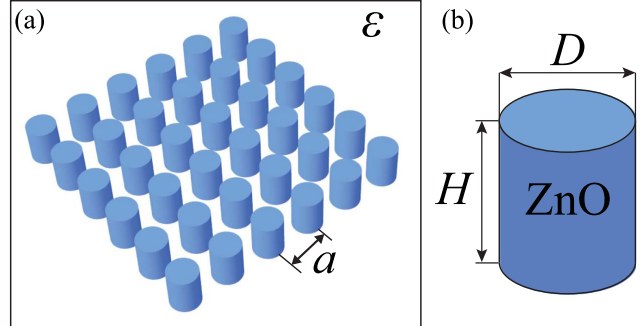

Fig. 1. (a) Sketch of the structure consisting of the square pattern of $\mathrm{ZnO}$ cylinders with a period $a$ in the dielectric homogeneous medium $\varepsilon$. (b) Unit cell representing a $\mathrm{ZnO}$ cylinder of diameter $\mathrm{D}$ and height $\mathrm{H}$.

Huygens' metasurface has been recently demonstrated at the telecommunication wavelength [34]. The operational principle of Huygens' surfaces is based on the degeneracy of the electric and magnetic dipole resonances in the far-field, but they are not configured to achieve the degeneracy of the surface or guided waves spectra.

In this work, we demonstrate the broadband TE and TM guided modes degeneracy with the periodic subwavelength pattern of $\mathrm{ZnO}$ cylinders. We show that the degeneracy can be achieved via tuning the period of the structure and the related cylinder dimensions. These results can be conveniently scaled to any frequency range in the near IR following the rules adopted for photonic crystals. We analyze the dependence of the guided modes dispersion and degeneracy breaking on the propagation direction due to the nonlocality and the effect of the substrate. Besides, we offer a special design of a $\mathrm{ZnO}$ structure on the aluminum-doped zinc oxide (AZO) substrate, which can potentially support the guided modes degeneracy in the vicinity of the telecommunication wavelength $1.55 \mu \mathrm{m}$. Finally, we propose the conceptually new practical application based on the guided modes degeneracy, namely linear-to-circular waveguide polarizer.

\section{RESULTS AND DISCUSSION}

\section{A. Broadband Polarization Degeneracy of Guided Modes}

In order to implement the broadband guided modes degeneracy for the near-IR range we choose $\mathrm{ZnO}$ nanocylinders in a homogeneous dielectric medium since they can be potentially fabricated with high quality [21], and they allow to configure dispersion via controlling their aspect ratio. We calculate the guided modes dispersion numerically by using the Eigenmode Solver of the CST Microwave Studio. We assume that the refractive index of $\mathrm{ZnO}$ is dispersionless, lossless and equals 1.95 since its dispersion changes very smoothly for the studied wavelength range ( $n=1.95 \pm 0.03$ in the frequency range from 140 to $430 \mathrm{THz}$ [35]).

We found that optimized diameter $D$ and height $H$ of the $\mathrm{ZnO}$ cylinder surrounded by air $(\varepsilon=1)$ should be $0.64 a$ and $0.825 a$, respectively, where $a$ is the period of the structure (see Fig. 1). It leads to the almost ideal coincidence of TE- and TM-polarized guided modes dispersion for the frequencies up to $0.42 c / a$ in the dimensionless units [Fig. 2(a)]. Since the
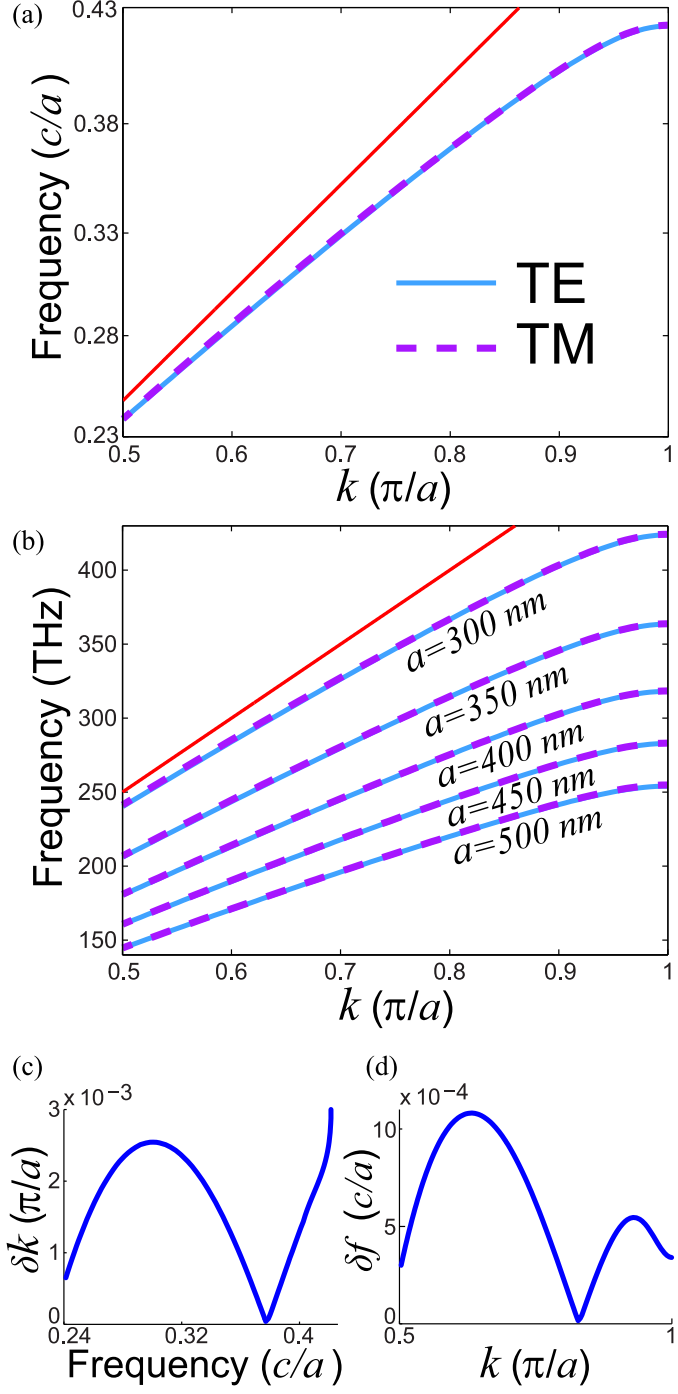

Fig. 2. Dependence of TE (cyan lines) and TM (purple lines) guided waves frequency on the wavevector within first Brillouin zone (a) in dimensionless frequency units $c / a$ and (b) in THz frequency units. Red line corresponds to the light line in the air [subfigure (b): light line is for $a=300 \mathrm{~nm}$ ]. (c) Dependence of the difference between the values of the wavevector of TE and TM modes on the frequency in the dimensionless units. (d) Dependence of the difference between the values of the frequency of TM and TE modes on the wavevector values in the dimensionless units.

cylinder dimensions are directly connected to the period of the structure, we can scale the geometric parameters to address the desired operation frequency range. One can see that the maximum frequency can change from $254 \mathrm{THz}$ for $a=500 \mathrm{~nm}$ to $424 \mathrm{THz}$ for $a=300 \mathrm{~nm}$ [Fig. 2(b)]. The appropriate difference between the wavevectors values of the TE and TM modes $\delta k$ does not exceed $0.003 \pi / a$ as shown in Fig. 2(c). It means that achieving a phase shift up to $0.3 \pi$ requires the maximum structure size up to $100 \times 100$ unit cells. The increase of this difference in the vicinity of the first Brillouin zone boundary is related to almost horizontal disposition of the dispersion curves. However, the dispersion degeneracy is still very high, which is confirmed by the difference between the values of the frequency of both modes [Fig. 2(d)]. 


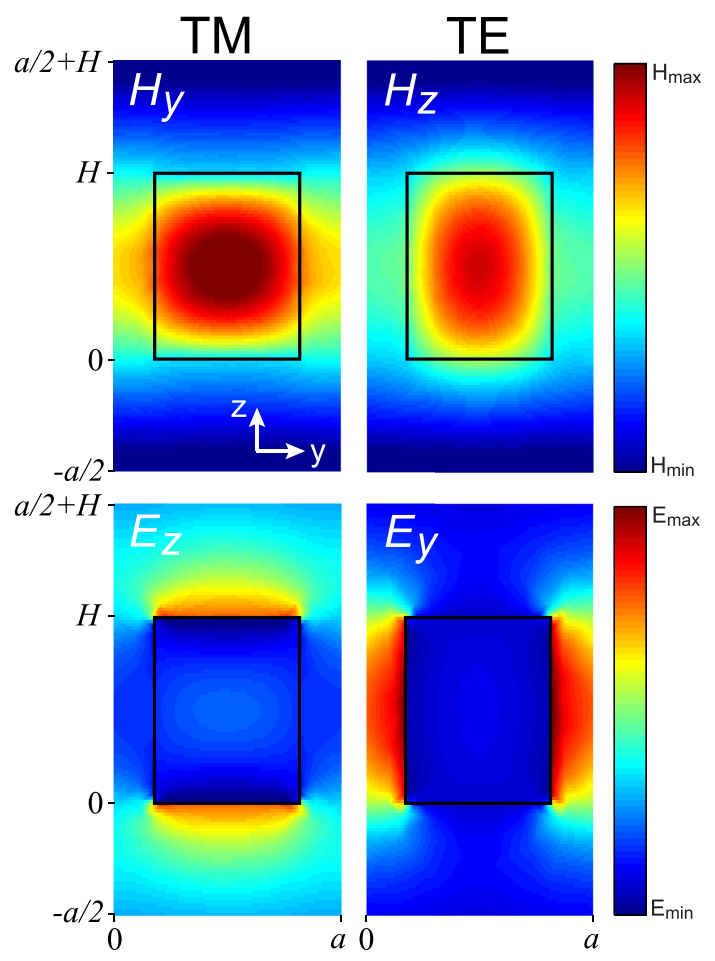

Fig. 3. Distribution of the electric and magnetic fields for TM- and TEpolarized guided modes for the structure, depicted in Fig. 1 with period $a=$ $450 \mathrm{~nm}$, at the wavelength 1547 and $1553 \mathrm{~nm}$, respectively, corresponding to $k=0.614 \pi / a$.

According to the dispersion diagrams [Fig. 2(b)] the structure is nothing else but a photonic crystal supporting the indexguided modes below the first bandgap. The eigenmodes can be classified as the guided waves of TM and TE polarizations. The spatial distribution of the electric and magnetic fields for both modes in the vicinity of the telecommunication wavelength is shown in Fig. 3. The amplitudes of the electromagnetic fields are approximately the same for both polarizations.

\section{B. Dependence on the Propagation Angles}

The main principle of the guided modes dispersion coincidence is the same as for the Huygens surfaces - the overlapping of electric and magnetic resonances of the structure [9]. Effective electric $\hat{\alpha}_{e}^{\text {eff }}$ and magnetic $\hat{\alpha}_{m}^{\text {eff }}$ polarizabilities of this periodic structure result from two contributions - the polarizability of a single cylinder $\hat{\alpha}_{0}(\omega)$ and the non-local interaction between the different cylinders $\hat{C}(\omega, \mathbf{k})$ :

$$
\begin{aligned}
& \hat{\alpha}_{i}^{\text {eff }}(\omega, \mathbf{k})=\hat{\alpha}_{0 i}(\omega)+\hat{C}_{i}(\omega, \mathbf{k}), \\
& i=e \text { (electric), } m \text { (magnetic). }
\end{aligned}
$$

The first term depends on the cylinder dimensions and material parameters, while the second one is characterized by the mutual arrangement of the cylinders. In contrast to conventional Huygens' metasurfaces, we investigate the eigenmodes under the light line, i.e. with the wavevectors larger than ones for light in free space. It means that interaction term in our case has greater impact comparing to Huygens' surfaces. Unlike the

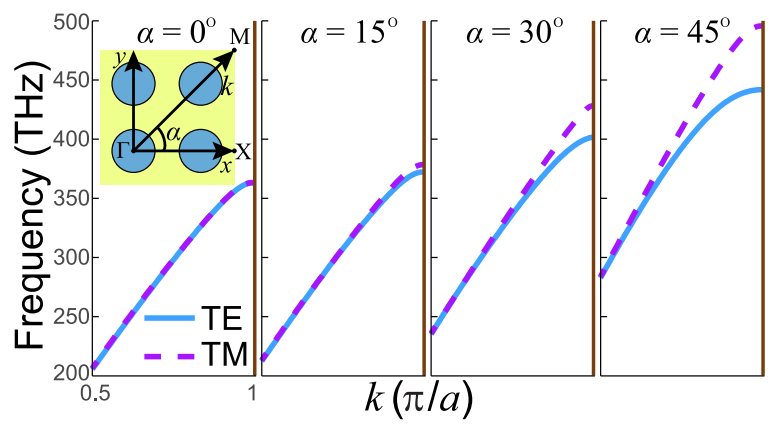

Fig. 4. Dispersion of the guided waves supported by the structure depicted in Fig. 1 for the different propagation angles $\alpha=0^{\circ}, 15^{\circ}, 30^{\circ}, 45^{\circ}$. Vertical brown lines correspond to the the first Brillouin zone boundary.
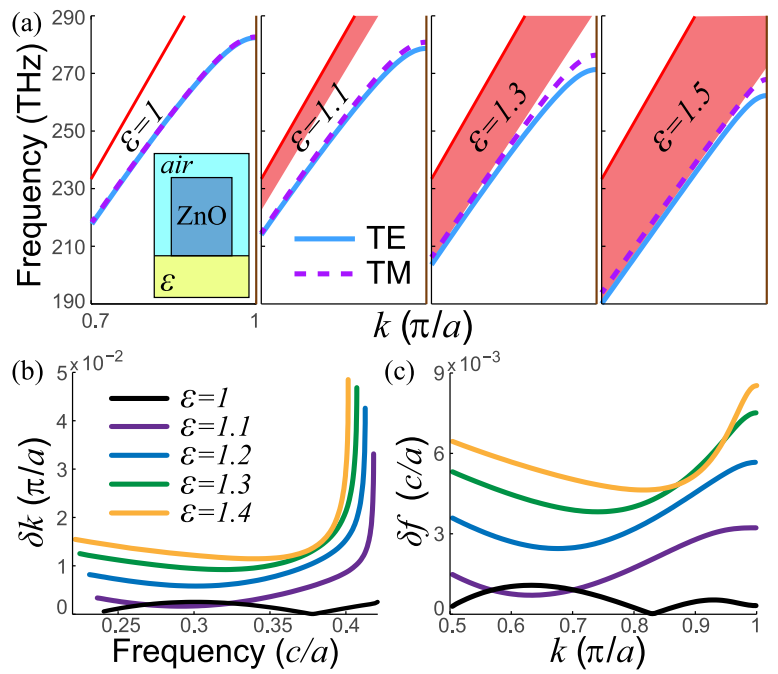

Fig. 5. (a) Dispersion of the guided waves supported by the structure depicted in the insert for the different substrate permittivity values $\varepsilon=1 ; 1.1 ; 1.3 ; 1.5$. Red line corresponds to the light line in the air. The filled pattern corresponds to the area between the light line in the air and in the substrate. The wavevector values change from 0.7 to 1 in $\pi / a$ units within the first Brillouin zone. Vertical brown lines correspond to the first Brillouin zone boundary. Dependences of the difference between the values of the wavevector of TE and TM modes on the frequency (b) and the difference between the values of the frequency of TM and TE modes on the wavevector values (c) for the different substrate permittivities are given below.

polarizability of the single cylinder, the interaction term is not radially symmetric and possesses the in-plane anisotropy. It can be explained by the different distances between the neighboring cylinders for the different directions and, therefore, it results in the different effective index. As a consequence, dispersion of guided waves depends on the propagation angle as well, which is shown in Fig. 4. One can see that the degeneracy is lifted as the degree of the anisotropy increases. The propagation angle $\alpha=45^{\circ}$ corresponds to the highest degree of anisotropy. So, the proposed design based on the $\mathrm{ZnO}$ cylinders offers the TE and TM guided modes degeneracy within full frequency range only in the limited range of the propagation angles.

\section{Role of Substrate}

It is necessary to analyze the effect of the substrate on the eigenmodes spectrum to suggest the practical implementation 


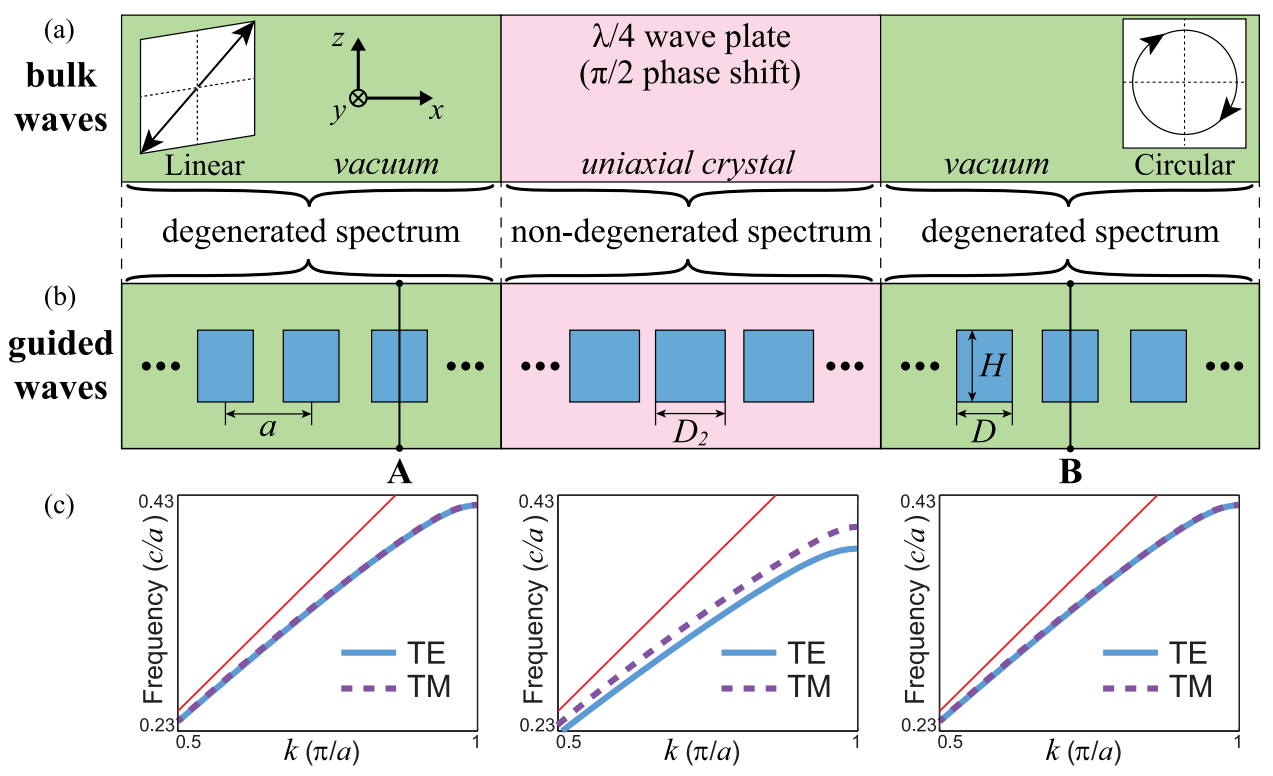

Fig. 6. The operational principle of a linear-to-circular polarizer based on the lifting of polarization degeneracy for (a) bulk waves (quarter-wave plate) and (b) guided waves (waveguide polarizer). The cylinder parameters are the following: $a=450 \mathrm{~nm}, H=0.825 a, D=0.64 a, D_{2}=0.8 a$. (c) The dispersions of TE- and TM-polarized guided modes for degenerated and non-degenerated regions of the photonic crystal waveguide. The guided waves propagate along the $x$-direction.

possibility. At first, the substrate changes the effective refractive index differently for TE and TM modes, which can lead to the fast degeneracy breaking. Secondly, with the presence of the substrate we have an asymmetric waveguide which can support guided modes only if the dielectric contrast or nanoparticles thickness are big enough. Thus, the emergence of the substrate leads to the asymmetric problem and decreases the permittivity contrast between the scatterer and the environment, which can result in the degeneracy breaking and eigenmodes vanishing. Figure 5(a) shows the guided modes dispersion for the different values of the substrate permittivity. One can observe the degeneracy breaking with the increase of the substrate permittivity [Figs. 5(b)-5(c)] and strong delocalization of the guided waves. The resonant frequency decreases due to the effective permittivity increase.

It is worth noting that the substrate does not have a significant impact for the high-index materials such as silicon. It is possible to design silicon nanocylinders on the fused silica substrate (in this case, silicon particle permittivity is approximately 6 times higher than substrate's one) in order to achieve the broadband degeneracy. Polarization degeneracy in a narrow band for silicon-on-insulator structure has been reported in Ref. [36]. For the $\mathrm{ZnO}$ nanocylinders the substrate leads to the degeneracy breaking. Nevertheless, the practical design can be implemented by using optically transparent artificial structures exhibiting refractive index close to one, for instance, heavily doped oxide semiconductors such as indium tin oxide (ITO) and aluminumdoped zinc oxide (AZO) [37], [38]. Specifically, we claim that it is possible to achieve the guided waves degeneracy ( $\delta k$ is around $0.002 \pi / a$ ) with the AZO substrate, described in the Ref. [39], namely $\varepsilon \approx 1.38+0.28 i$ at $1.55 \mu \mathrm{m}$, in the vicinity of the telecommunication wavelength by appropriately designing the $\mathrm{ZnO}$ cylinders as follows: $D=0.72 a ; H=0.88 a$.

\section{Waveguide Polarizer}

Discovering polarization degrees of freedom for guided and surface waves leads to a number of potential applications in light technologies. In this Section we analyze the opportunity to create a polarizer for guided waves similar to the one for bulk waves. Specifically, we elaborate the concept of a quarter-wave plate (linear-to-circular polarizer) for guided waves.

The operational principle of a conventional quarter-wave plate is based on the withdrawal of the polarization degeneracy within a finite propagation region. Initially, an electromagnetic wave of linear polarization propagates in vacuum supporting the polarization degenerated spectrum. Then, it enters an uniaxial crystal breaking the degeneracy due to the medium anisotropy. The phase difference between two modes reaches $\pi / 2$, when wave is coming out from the uniaxial crystal to vacuum, i.e. its polarization becomes circular (or elliptical). This concept is sketched in Fig. 6(a). We propose to implement the analogue of the quarter-wave plate for guided waves, 'waveguide polarizer', by using two types of waveguides supporting degenerated and fairly non-degenerated spectra as the analogues of vacuum and uniaxial crystal, respectively. The waveguide with the degenerated spectrum has been investigated above, while the waveguide with lifted degeneracy can be obtained by changing the cylinders aspect ratio [Fig. 6(b)]. The dispersion of TE- and TM-polarized modes in both waveguides is shown in Fig. 6(c).

Figure 7 shows the electric field distributions before [crosssection A in Fig. 6(b)] and after [cross-section B in Fig. 6(b)] the waveguide polarizer. It unambiguously demonstrates the polarization transformation of guided waves from linear (at the input) to the elliptical one (at the output). The polarization device proposed exhibits great opportunities for conceptually new integrated circuits and on-chip devices using the polarization 


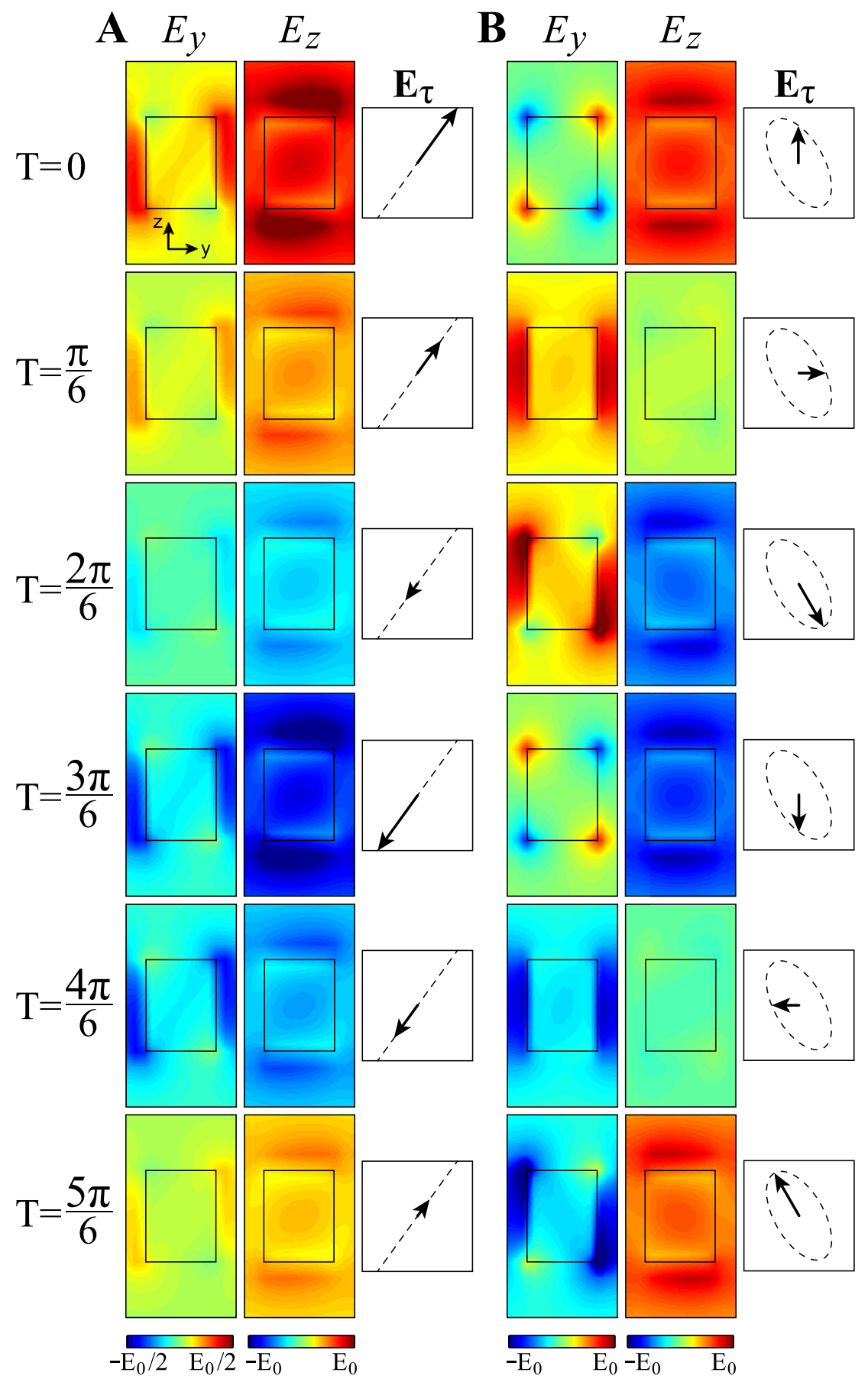

Fig. 7. The distributions of tangential electric field components $\operatorname{Re}\left[E_{j} e^{i T}\right](j=y, z)$ in YZ-plane before (A) and after (B) the waveguide polarizer for different times $T$ at $250 \mathrm{THz}$. The color bar changes from negative (blue) to positive (red) values, which allows to define the direction and absolute value of the tangential electric field vector $\mathbf{E}_{\tau}$ (right columns).

degree of freedom of localized waves for the optical encoding, transfer and processing information.

\section{CONCLUSION}

To conclude, we have shown that a photonic crystal structure based on a square lattice of the high-index dielectric cylinders in the homogeneous low-index dielectric medium supports the broadband TE and TM guided modes degeneracy with high accuracy. We have calculated the appropriate design for the $\mathrm{ZnO}$ cylinders surrounded by air. This effect persists only in the limited range of the propagation angles as a consequence of the strong nonlocal impact. The substrate with permittivity greater than 1.3 breaks the degeneracy and leads to the guided waves vanishing. However, we have offered the practical design based on the $\mathrm{ZnO}$ cylinders on the AZO substrate in order to achieve the degeneracy in the vicinity of the telecommunication wavelength. Finally, the concept of the waveguide polarizer based on the polarization-degenerated eigenmodes spectrum has been presented. These results can be easily extended on a single-roll structure, other shapes and lattice types of the $\mathrm{ZnO}$ 
pattern, holes in the $\mathrm{ZnO}$ matrix and other dielectric materials such as silicon, titanium dioxide, sapphire, etc. We consider this work as a significant milestone towards the development and creation of the flat optics and on-chip devices.

\section{ACKNOWLEDGMENT}

The authors would like to thank Yu. S. Kivshar for the fruitful discussions.

\section{REFERENCES}

[1] S. Jahani and Z. Jacob, "All-dielectric metamaterials," Nature Nanotechnol., vol. 11, no. 1, pp. 23-36, 2016.

[2] D. G. Baranov et al., "All-dielectric nanophotonics: The quest for better materials and fabrication techniques," Optica, vol. 4, no. 7, pp. 814-825, 2017.

[3] A. I. Kuznetsov, A. E. Miroshnichenko, Y. H. Fu, J. Zhang, and B. Luk' yanchuk, "Magnetic light," Sci. Rep., vol. 2, 2012, Art. no. 492.

[4] A. B. Evlyukhin et al., "Demonstration of magnetic dipole resonances of dielectric nanospheres in the visible region," Nano Lett., vol. 12, no. 7, pp. 3749-3755, 2012.

[5] A. I. Kuznetsov, A. E. Miroshnichenko, M. L. Brongersma, Y. S. Kivshar, and B. Luk'yanchuk, "Optically resonant dielectric nanostructures," Science, vol. 354, no. 6314, 2016, Art. no. aag2472.

[6] Y. Kivshar and A. Miroshnichenko, "Meta-optics with Mie resonances," Opt. Photon. News, vol. 28, no. 1, pp. 24-31, 2017.

[7] A. E. Krasnok, A. E. Miroshnichenko, P. A. Belov, and Y. S. Kivshar, "Alldielectric optical nanoantennas," Opt. Express, vol. 20, no. 18, pp. 2059920604, 2012.

[8] C. Pfeiffer and A. Grbic, "Metamaterial Huygens' surfaces: Tailoring wave fronts with reflectionless sheets," Phys. Rev. Lett., vol. 110, no. 19, 2013, Art. no. 197401

[9] M. Decker et al., "High-efficiency dielectric Huygens' surfaces," Adv. Opt. Mater, vol. 3, no. 6, pp. 813-820, 2015.

[10] A. Krasnok et al., "Demonstration of the enhanced Purcell factor in all-dielectric structures," Appl. Phys. Lett., vol. 108, no. 21, 2016, Art. no. 211105 .

[11] A. E. Miroshnichenko et al., "Nonradiating anapole modes in dielectric nanoparticles," Nature Commun., vol. 6, 2015, Art. no. 8069.

[12] B. Luk'yanchuk, R. Paniagua-Domínguez, A. I. Kuznetsov, A. E. Miroshnichenko, and Y. S. Kivshar, "Hybrid anapole modes of highindex dielectric nanoparticles," Phys. Rev. A, vol. 95, no. 6, 2017, Art. no. 063820.

[13] D. Lin, P. Fan, E. Hasman, and M. L. Brongersma, "Dielectric gradient metasurface optical elements," Science, vol. 345, no. 6194, pp. 298-302, 2014.

[14] A. Arbabi, Y. Horie, M. Bagheri, and A. Faraon, "Dielectric metasurfaces for complete control of phase and polarization with subwavelength spatial resolution and high transmission," Nature Nanotechnol., vol. 10, no. 11, pp. 937-943, 2015.

[15] O. Takayama, D. Artigas, and L. Torner, "Lossless directional guiding of light in dielectric nanosheets using Dyakonov surface waves," Nature Nanotechnol., vol. 9, no. 6, pp. 419-424, 2014.

[16] O. Takayama, A. Bogdanov, and A. V. Lavrinenko, "Photonic surface waves on metamaterial interfaces," J. Phys., Condens. Matter, vol. 29 , no. 46, 2017, Art. no. 463001

[17] C. Jagadish and S. J. Pearton, Zinc Oxide Bulk, Thin Films and Nanostructures: Processing, Properties, and Applications. Amsterdam, The Netherlands: Elsevier, 2011.

[18] W. J. Beek, M. M. Wienk, and R. A. Janssen, "Efficient hybrid solar cells from zinc oxide nanoparticles and a conjugated polymer," Adv. Mater. vol. 16, no. 12, pp. 1009-1013, 2004.

[19] E. Comini, "Metal oxide nano-crystals for gas sensing," Analytica Chimica Acta, vol. 568, no. 1/2, pp. 28-40, 2006.

[20] Z. L. Wang, "Zinc oxide nanostructures: growth, properties and applications," J. Phys., Condens. Matter, vol. 16, no. 25, pp. R829-R858, 2004.

[21] S. Okamoto et al., "Fabrication of single-crystalline microspheres with high sphericity from anisotropic materials," Sci. Rep., vol. 4, 2014, Art. no. 5186.

[22] X. Huang, Y. Lai, Z. H. Hang, H. Zheng, and C. Chan, "Dirac cones induced by accidental degeneracy in photonic crystals and zero-refractiveindex materials," Nature Mater., vol. 10, no. 8, pp. 582-586, 2011.
[23] A. Pors, O. Albrektsen, I. P. Radko, and S. I. Bozhevolnyi, "Gap plasmonbased metasurfaces for total control of reflected light," Sci. Rep., vol. 3, 2013, Art. no. 2155.

[24] O. Y. Yermakov et al., "Spin control of light with hyperbolic metasurfaces," Phys. Rev. B, vol. 94, no. 7, 2016, Art. no. 075446.

[25] L. Carroll, D. Gerace, I. Cristiani, and L. C. Andreani, "Optimizing polarization-diversity couplers for Si-photonics: reaching the $-1 \mathrm{~dB}$ coupling efficiency threshold," Opt. Express, vol. 22, no. 12, pp. 14769-14781, 2014.

[26] J. Zou, Y. Yu, and X. Zhang, "Single step etched two dimensional grating coupler based on the SOI platform," Opt. Express, vol. 23, no. 25, pp. 32490-32495, 2015.

[27] J. Zou, Y. Yu, and X. Zhang, "Two-dimensional grating coupler with a low polarization dependent loss of $0.25 \mathrm{~dB}$ covering the C-band," Opt. Lett., vol. 41, no. 18, pp. 4206-4209, 2016.

[28] G. Pellegrini, M. Finazzi, M. Celebrano, L. Duò, and P. Biagioni, "Chiral surface waves for enhanced circular dichroism," Phys. Rev. B, vol. 95, no. 24, 2017, Art. no. 241402.

[29] G. Pellegrini, M. Finazzi, M. Celebrano, L. Duò, and P. Biagioni, "Surfaceenhanced chiroptical spectroscopy with superchiral surface waves," Chirality, vol. 30, no. 7, pp. 883-889, 2018.

[30] Y. F. Yu et al., "High-transmission dielectric metasurface with $2 \pi$ phase control at visible wavelengths," Laser Photon. Rev., vol. 9, no. 4, pp. 412 418, 2015.

[31] K. E. Chong et al., "Efficient polarization-insensitive complex wavefront control using Huygens' metasurfaces based on dielectric resonant metaatoms," ACS Photon., vol. 3, no. 4, pp. 514-519, 2016.

[32] V. Asadchy et al., "Broadband reflectionless metasheets: frequencyselective transmission and perfect absorption," Phys. Rev. X, vol. 5, no. 3, 2015, Art. no. 031005

[33] M. Londoño, A. Sayanskiy, J. L. Araque-Quijano, S. B. Glybovski, and J. D. Baena, "Broadband Huygens' metasurface based on hybrid resonances," Phys. Rev. Appl., vol. 10, 2018, Art. no. 034026.

[34] C. Pfeiffer et al., "Efficient light bending with isotropic metamaterial Huygens' surfaces," Nano Lett., vol. 14, no. 5, pp. 2491-2497, 2014.

[35] W. Bond, "Measurement of the refractive indices of several crystals," $J$. Appl. Phys., vol. 36, no. 5, pp. 1674-1677, 1965.

[36] P. J. Bock et al., "Subwavelength grating periodic structures in silicon-oninsulator: A new type of microphotonic waveguide," Opt. Express, vol. 18 , no. 19, pp. 20251-20262, 2010.

[37] M. Noginov et al., "Transparent conductive oxides: Plasmonic materials for telecom wavelengths," Appl. Phys. Lett., vol. 99, no. 2, 2011, Art. no. 021101

[38] G. V. Naik, J. Kim, and A. Boltasseva, "Oxides and nitrides as alternative plasmonic materials in the optical range," Opt. Mater. Express, vol. 1, no. 6, pp. 1090-1099, 2011.

[39] G. V. Naik, J. Liu, A. V. Kildishev, V. M. Shalaev, and A. Boltasseva, "Demonstration of $\mathrm{Al}: \mathrm{ZnO}$ as a plasmonic component for near-infrared metamaterials," Proc. Nat. Acad. Sci., vol. 109, no. 23, pp. 8834-8838, 2012.

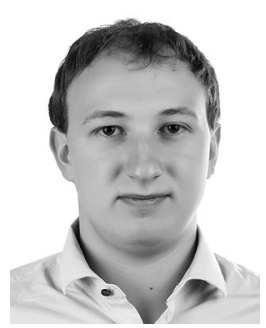

Oleh Y. Yermakov (S'18) was born in Kharkiv, Ukraine, in 1993. He received the B.Sc. degree (cum laude) in Applied Physics from the Kharkiv National University, Ukraine, in 2014, and the M.Sc. degree in Photonics and Optical Information Technologies from ITMO University, Saint Petersburg, Russia in 2016, where he is currently pursuing the Ph.D. degree in Optics

Since 2014, he has been with the International Research Center for Nanophotonics and Metamaterials, ITMO University. His research interests include analytical and numerical modeling of metamaterials and photonic crystals, metamaterial characterization, metasurfaces, near-field optics, electromagnetic surface waves, and all-dielectric photonics.

Mr. Yermakov is a Member of the International Society for Optical Engineers (SPIE), Bellingham, WA, USA, the Optical Society of America, Washington, DC, USA. He received the scholarship for students in theoretical physics from the Dynasty Foundation in 2015, the SPIE Travel Scholarship in 2016, and the individual research grant "Ph.D. Student" from the Foundation for the Advancement of Theoretical Physics and Mathematics "BASIS" in 2017. 


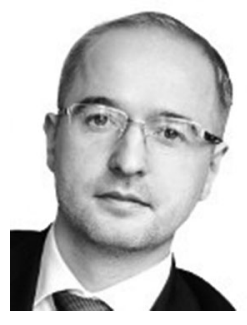

Andrey A. Bogdanov was born in Saint Petersburg, Russia, in 1986. He received the B.Sc. and M.Sc. degrees in Solid State Physics from the Saint Petersburg State Polytechnical University, Saint Petersburg, Russia, in 2007 and 2009, respectively, and the Ph.D. degree in Physics of Semiconductors from the Ioffe Institute, Saint Petersburg, Russia, in 2013.

In 2014, he got a Postdoc position with the International Research Center for Nanophotonics and Metamaterials, ITMO University, Saint Petersburg, Russia, where he is currently an Assistant Professor. His research interests include the theory of surface waves, bound states in the continuum, optomechanics, semiconductor lasers, nonlinear optics, and photonic structures.

Dr. Bogdanov is the Chair of the Annual Doctoral Summer School on Nanophotonics and Metamaterials.

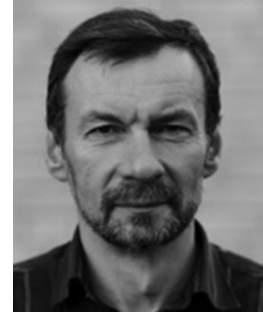

Andrei V. Lavrinenko received the M.S., Ph.D., and D.Sci. degrees from the Belarusian State University (BSU), Minsk, Belarus, in 1982, 1989, and 2004, respectively.

From 1990 to 2004, he was an Assistant Professor and Associate Professor with the Department of Physics, BSU. Since 2004, he has been an Associate Professor with the Department of Photonics Engineering, Technical University of Denmark, Kongens Lyngby, Denmark. Since 2008, he has been leading the Metamaterials Group of this department. He is the author of five textbooks, ten book chapters, and more than 180 journal papers. His research interests include metamaterials, plasmonics, photonic crystals, quasicrystals and photonic circuits, slow light, and numerical methods in electromagnetics and photonics. 